Jennifer Fernandes Pamplona

\title{
Udwadia-Kalaba Method for Obtaining the Dynamics of Constrained Systems
}

\author{
Graduation Project
}

Mechanical Engineering Department

Advisor: Roberta Lima 


\section{Acknowledgements}

I would like to use this section to express my gratitude to the following parties, for without them, this project would not have been possible.

First, I wish to express my sincere gratitude to my supervisor, Professor Roberta Lima, for her patience, insightful comments and helpful information that have helped me tremendously at all times in my research of this project.

I would like to thank PUC-Rio and all its faculty who contributed directly and indirectly to the completion of this project.

I wish to thank my mom, Alessandra Fernandes, who taught me that knowledge is the most valuable thing we can have, as no one has the power to take it away from us. Without her, my entire academic trajectory would be impossible.

To my father Petterson Medeiros (in memoriam), who, even not being present at this moment, taught me values that I carry with me for my whole life.

To my sister, Stephanie Pamplona, for believing in me and cheering for me.

To my cousins, Marcelly Vieira and Lucas Meirinho, for their unconditional support and for understanding my moments of absence during this year.

To my grandfather, Carlos Fernando Pamplona, who has always been my source of inspiration.

To my college friends for making these five years of graduation lighter and complementing my education with experiences and new knowledge.

Finally, thanks to everyone who contributed directly or indirectly to my graduation. 


\section{Resumo}

\section{Método de Udwadia-Kalaba para Obter a dinâmica de sistemas com Vín- culos}

Neste trabalho é apresentada uma estrutura geral para a equação explícita de movimento para sistemas mecânicos, desenvolvida por Udwadia e Kalaba, que estão sujeitos a vínculos holonômicos e não holonômicos. Essas novas equações levam a uma visão básica simples e nova da mecânica lagrangiana. A equação Udwadia-Kalaba pode ser derivada através do princípio de Gauss, princípio de d'Alembert ou princípio de Alembert estendido. Uma vez explicadas as equações dinâmicas, será demonstrada a aplicabilidade do método em alguns exemplos de sistemas mecânicos em problemas de engenharia, como um pêndulo com um suporte móvel. Outro exemplo será uma partícula que é restringida a se mover ao longo de uma trajetória elíptica. Os resultados fornecem ideias mais profundas sobre o caráter fundamental do movimento restrito em sistemas mecânicos em geral. Além disso, a comparação da equação de UdwadiaKalaba com a equação de Newton-Euler é feita.

\section{Keywords}

Udwadia-Kalaba; Vínculos; Equação fundamental; Sistemas Mecânicos; Inversa Generalizada de Moore-Penrose. 


\section{Abstract}

\section{Udwadia-Kalaba Method for Obtaining The Dynamics of Constrained Systems}

In this project is presented a general structure fot the explicit equation of motion for constrained mechanical systems, developed by Udwadia and Kalaba, which are subjected to holonomic and non-holonomic constraints. These new equations lead to a simple and new fundamental view of lagrangian mechanics. The Udwadia-Kalaba equation can be derived via the Gauss's principle, d'Alembert's principle or extended d'Alembert's principle. Once the dynamic equations are explained, shall be demonstrated the applicability of the method in some examples of mechanical systems in engineering problems, as a pendulum with a moving support. Another example will be a particle that is constrained to move along an elliptical trajectory. The results provide deeper insights into the fundamental character of constrained motion in general mechanical systems. Furthermore, a comparison of Udwadia-Kalaba Equation with Newton-Euler Equation is made.

\section{Keywords}

Udwadia-Kalaba; Constraint; Fundamental Equation; Mechanical Systems; Moore-Penrose Generalized Inverse. 


\section{Contents}

1 Introduction $\quad 2$

2 Udwadia-Kalaba Method 4

2.1 Important Concepts . . . . . . . . . . . . . . . . . 4

2.1.1 Generalized Inverse of a Matrix . . . . . . . . . . 4

2.2 Moore-Penrose Generalized Inverse . . . . . . . . . . . . 4

2.2.1 Consistency of the System Equation . . . . . . . . . 7

2.2.2 General Solution . . . . . . . . . . . . . . . 7

2.3 The Fundamental Equation . . . . . . . . . . . . . . 8

2.3.1 Constraints and Types of Constraints . . . . . . . 8

2.3.2 Gauss's Principle . . . . . . . . . . . . . . . . . 12

2.3.3 Fundamental Equation . . . . . . . . . . . . . 14

2.3.4 The Force of Constraint . . . . . . . . . . . . 15

3 Example of constrained motions $\quad 17$

3.1 Example 1: Scleronomic constraint . . . . . . . . . . . . 17

3.2 Example 2: Rheonomic constraint . . . . . . . . . . . 17

3.3 Example 3: Fundamental Equation Applied 1 . . . . . . . . . 17

3.4 Example 4: Fundamental Equation Applied 2 . . . . . . . . 23

4 Conclusions $\quad 28$

5 Bibliography 29

$\begin{array}{ll}\text { Appendices } & 31\end{array}$

A Matlab Script 31

B Mathematica Script 33 


\section{List of Figures}

1 Coupled Duffing's Oscillator . . . . . . . . . . . . . 18

2 Response of the system without constraint forces . . . . . . 20

3 Response of the system with constraint forces . . . . . . . . 21

4 Figure illustrating the effect of the constraint force . . . . . 22

5 The force of constraint acting on the two masses . . . . . . . 22

6 Numerical error due to the accuracy of the integration scheme . 23

7 Two-link pendulum . . . . . . . . . . . . . . . 24

8 Response of doubly constrained pendulum showing nonlinear behavior ..................... 26

9 Out-of-plane motion of doubly constrained pendulum . . . . . 26

10 Trajectory of the constrained motion of the pendulum bob . . . . 27 


\section{Introduction}

The determination of equations of motion for constrained mechanical systems is one of the central problems in analytical dynamics [2]. Up to 1990, all existing methods of analytical mechanics were based on some auxiliary variables, as Newton-Euler mechanic equations and Lagrange equations. In 1992, Udwadia and Kalaba proposed a new approach to this problem in classical mechanics. This approach provides an analytical expression of the equation of motion for constrained mechanical systems, where the constraints can be holonomic and/or nonholonomic. Since the Udwadia-Kalaba Equation of motion for constrained mechanical seems to be the one of the simplest and the most comprehensive forms of determining the equations of constraints, it is worth giving a deeper look.

Constrained motion results when an object is forced to move in a restricted way. When a particle is compelled to move along a particularly given path under the action of external impressed forces. The restrictions imposed on the motion or position or both of a system of particles are known as constraints. The forces that the constraining object exerts on the object to make it follow the constraints of movement are known as Constraint forces.

In the project, the constraints will be limited to the concept of holonomic and nonholonomic constraints, although the theory is valid for a much larger class of constraints. Holonomic constraints are the ones, where the constraints are on the position or configuration of a system of particles. If the holonomic constraint does not have time explicitly in it, it is called a scleronomic constraint; else it is called rheonomic. Nonholonomic systems are systems where the velocities (magnitude and/or direction) and other derivatives of the position are constrained. However, even though in a holonomic constraint velocities do not directly appear, constraints are place on the velocities of the system of particles, because the holonomic constraint must be satisfied at all times.

Over the last 200 years, a considerable body of literature developed around the determination of the equations of motion for constrained systems. Many of these contributions have come from physicists and mathematicians like Lagrange, Euler, Gauss and Gibbs. In this project, will be shown that these constraints can be handled with equal ease by the methods of Udwadia and Kalaba. Contrary to the other methods, this one does not require any concepts to handle nonintegrable 
as opposed to integrable constraints. This method encompasses the entirety of Lagrangian mechanics. To understand in depth the relations stated, it is needed a greater familiarity with some elements of linear algebra. Therefore, in this project will also be presented some of the fundamentals of linear algebra, as the Moore-Penrose generalized inverse and Gauss Principle. 


\section{Udwadia-Kalaba Method}

\subsection{Important Concepts}

To understand in depth the process of obtaining the dynamics of constrained systems, a greater familiarity with some elements of linear algebra is needed. Hence, in this section some fundamentals of linear algebra are presented.

\subsubsection{Generalized Inverse of a Matrix}

Consider this set of equations

$$
A x=b .
$$

where $A$ is an $m$ by $n$ matrix, $x$ is an $n$ by 1 vector. If $m=n$ and the matrix $A$ is a nonsingular matrix, then the unique solution of this set of equations is obtained as $x=A^{-1} b$. However, as will be shown in a future section, the matrix $A$ will usually be non-square. Therefore, the notion of the inverse of a matrix need to be generalized, developing a "generalized" inverse which will handle non-square matrices as well as square matrices that are singular.

Specifically, it will be defined three types of generalized inverses: the Ginverse, the L-inverse and the Moore-Penrose inverse. The G-inverse is better suited to solve a consistent set of linear equations of the form $A x=b$, where $\mathrm{A}$ is no longer a square matrix. The L-inverse is suited when solving the least square problem of finding $\mathrm{x}$ so that $\|A x-b\|^{2}$ is minimised, again for a non-square matrix $A$. For the main purpose of this project, the Moore-Penrose inverse will be the most useful, since it is both a G-inverse and a L-inverse.

\subsection{Moore-Penrose Generalized Inverse}

Consider the $m$ by $n$ matrix $A$ which has rank $r$. Will be denoted the $n$ by $m$ matrix $A^{+}$, called Moore-Penrose (MP) inverse of the matrix A, if the matrix $A^{+}$ satisfies all the following four conditions:

1. $A A^{+} A=A$.

2. $A^{+} A A^{+}=A^{+}$. 
3. $A A^{+}=\left(A A^{+}\right)^{T}$, i.e., the matrix $A A^{+}$is symmetric.

4. $A^{+} A=\left(A^{+} A\right)^{T}$, i.e., the matrix $A^{+} A$ is symmetric.

In this project, these conditions will be called the Moore-Penrose conditions. Concerning the G-inverse matrix, consider the $m$ by $n$ matrix $A$ which has rank $r$. Any $n$ by $m$ matrix $A^{G}$ for which the first MP condition is satisfied is called a G-inverse of the matrix A. Thus the G-inverse of $\mathrm{A}$, denoted $A^{G}$, satisfies the condition

$$
A A^{G} A=A \text {. }
$$

Concerning the L-inverse matrix, consider the $m$ by $n$ matrix $A$ which has rank $r$. Any $n$ by $m$ matrix $A^{L}$ for which the first and third MP conditions are satisfied is called an L-inverse of the matrix A. Thus the L-inverse of A, denoted $A^{L}$, satisfies the two conditions

$$
A A^{L} A=A \text {. }
$$

and

$$
A A^{L}=\left(A A^{L}\right)^{T} .
$$

Notably, the L-inverse of the matrix $A$ must satisfy one additional condition which the G-inverse does not have to. Every L-inverse of the matrix $A$ is therefore automatically a G-inverse of $A$. Furthermore, there can be more than one G-inverse and more than one L-inverse of a given matrix $A$. Hence for any matrix $A$, there is a set of matrices which all satisfy equation (2), and therefore any one of these matrices is a G-inverse of $A$; similarly we have a set of matrices which are L-inverses of $A$. However, will be shown that the MP-inverse of any given matrix $\mathrm{A}$ is unique. Because of the way these matrices have been defined, any matrix which is an MP-inverse of $A$ is also an L-inverse of $A$, and any matrix which is an L-inverse of $A$ is also a G-inverse of $A$.

Consider two different MP-inverses, say $A_{1}^{+}$and $A_{2}^{+}$, of an $m$ by $n$ matrix $A$. It will be shown that if this is true then $A_{1}^{+}=A_{2}^{+}$, as stated in the previous paragraph. First, is shown that $A A_{1}^{+}=A A_{2}^{+}$and $A_{2}^{+} A=A_{1}^{+} A$.

Since $A_{1}^{+}$is an MP-inverse, $A=A A_{1}^{+} A$. Hence, multiplying on the right by $A_{2}^{+}$we get 


$$
A A_{2}^{+}=A A_{1}^{+} A A_{2}^{+} .
$$

However, by the MP-inverse conditions, $A A_{2}^{+}$is symmetric. Hence the right hand side of equation (5) must also be symmetric, and so $A A_{1}^{+} A A_{2}^{+}=\left(A A_{1}^{+} A A_{2}^{+}\right)^{T}$. Thus we get

$$
\begin{aligned}
A A_{2}^{+}=A A_{1}^{+} A A_{2}^{+} & =\left(A A_{1}^{+} A A_{2}^{+}\right)^{T} \\
& =\left(A A_{2}^{+}\right)^{T}\left(A A_{1}^{+}\right)^{T} \\
& =\left(A A_{2}^{+}\right)\left(A A_{1}^{+}\right) \\
& =\left(A A_{2}^{+} A\right) A_{1}^{+} \\
& =A A_{1}^{+}
\end{aligned}
$$

Notably, $\left(A A_{2}^{+}\right)^{T}=A A_{2}^{+}$because $A_{2}^{+}$is an MP-inverse and the third MP condition must therefore be satisfied.

Through some properties of the MP-inverses, some commonly occurring matrices are:

1.

$$
(c A)^{+}=\frac{1}{c} A^{+}
$$

where $c$ is a nonzero scalar

2. If $a$ is a non zero 1 by $n$ row vector, and $b$ is a nonzero $n$ by 1 column vector then,

$$
\begin{aligned}
& a^{+}=\frac{1}{a a^{T}} a^{+}, \\
& b^{+}=\frac{1}{b b^{T}} b^{+} .
\end{aligned}
$$

3. If $a$ is a nonzero 1 by $n$ vector, then

$$
\begin{gathered}
\left(a^{T} a\right)^{+}=\frac{1}{\left(a a^{T}\right)^{2}} a^{T} a, \\
\text { if }\left[\begin{array}{cc}
B & 0 \\
0 & C
\end{array}\right], \text { then } A^{+}=\left[\begin{array}{cc}
B^{+} & 0 \\
0 & C^{+}
\end{array}\right] .
\end{gathered}
$$

4. For any $m$ by $n$ matrix $A$, whatever its rank, the following relations hold: 


$$
\begin{aligned}
& A^{+}=A^{T}\left(A A^{T}\right)^{+} \\
& A^{+}=\left(A A^{T}\right)^{+} A^{T}
\end{aligned}
$$

5. If $\mathrm{A}$ has rows $i_{1}, i_{2}, \ldots ., i_{q}$ which are proportional, then these same columns in $A^{+}$will also be proportional. In particular if $q$ rows of $A$ are zero, then the corresponding $q$ columns of $A^{+}$are also zero.

\subsubsection{Consistency of the System Equation}

The system of equations given by equation (1) is consistent if and only if

$$
A A^{G} b=b
$$

where $A^{G}$ is any $\mathrm{G}$-inverse of $\mathrm{A}$

1. Assuming that the system is consistent and let the vector $x$ satisfy the system of equations. Then multiply $A x=b$ on the left by $A A^{G}$, we then get

$$
A A^{G} A x=A A^{G} b
$$

Using equation (2), the left hand side of equation (15) is simply $A A^{G} A x=$ $A x=b$. By setting the left hand side of (15) to the right hand side, we get

$$
b=A A^{G} b
$$

2. Now, assuming that $b=A A^{G} b$. Let $x=A^{G} b$. Substituting for this value of $\mathrm{x}$ in the equation (1), we get $A\left(A^{G} b\right)=b$. Hence $x=A^{G} b$ is the solution of the equation (1).

\subsubsection{General Solution}

Let $A$ be an $m$ by $n$ matrix. The general solution $x$ of the equation $A x=b$, assuming it exists, is

$$
x=A^{G} b+\left(I-A^{G} A\right) h
$$


where $h$ is any $n$ by 1 vector. $A^{G}$ is any G-inverse of A. Furthermore, every solution of $A x=b$ can be expressed in the form of equation (17) for some $n$ by 1 vector $h$.

Since the equation is consistent, by equation (14), $A A^{G} b=b$. To prove that $x$ is a solution of the equation, multiply equation (17) by $A$. This gives

$$
A x=A A^{G} b+A\left(I-A^{G} A\right) h=b+(A-A) h=b
$$

Suppose a solution $x$ to the equation $A x=b$. Since $x$ is a solution, $A x=b$, and hence $A^{G} A x=A^{G} b$, so that $0=A^{G} b-A^{G} A x$. Adding $x$ to both sides, what is left is $x=A^{G} b+\left(I-A^{G} A\right) x$, which is the form of equation (17). Hence the result.

\subsection{The Fundamental Equation}

The study will begin on a discussion on the nature of some elementary constraints, where it will be better explained the concepts of holonomic and nonholonomic, which were presented at the introduction. Next, the Gauss's principle will be discussed, which will give a clear description of the general nature of constrained motion in terms of the minimization of a function of the acceleration of the particles of a system. It is from this principle that the fundamental equation, which describes the dynamics of the constrained systems, will be obtained.

\subsubsection{Constraints and Types of Constraints}

The reason why the study of constraints is first introduced for the dynamical systems analysis is to understand constrained motion we must understand the nature and types of constraints that we will be dealing with in this project.

Holonomic Constraints: Consider a single particle of mass $m$ moving along a straight line which is aligned along the $\mathrm{X}$-axis of an inertial rectangular coordinate system. The equation of motion is usually written as:

$$
m \ddot{x}=F_{x}(x, \dot{x}, t),
$$

where $x(t)$ is the position of the particle along the line, measured from a fixed point on it, and $F_{x}(x, \dot{x}, t)$ is the impressed force acting on the particle. However, 
to fully describe the problem, the three equations of motion should be written as

$$
\begin{aligned}
& m \ddot{x}=F_{x}(x, \dot{x}, y, \dot{y}, z, \dot{z}, t), \\
& m \ddot{y}=F_{y}(x, \dot{x}, y, \dot{y}, z, \dot{z}, t), \\
& m \ddot{z}=F_{z}(x, \dot{x}, y, \dot{y}, z, \dot{z}, t),
\end{aligned}
$$

and add the constraints

$$
y(t)=0 \quad \text { and } \quad z(t)=0
$$

By differentiating these constraint equations, we obtain, $\dot{y}=\dot{z}=0$, and with one more differentiation yields $\ddot{y}=\ddot{z}=0$. Using the last two equations of the set of equations (20), they imply that $F_{y}(t)=F_{z}(t)=0$, and the three equations in the set of equations (20) reduce to the single equation

$$
m \ddot{x}=F_{x}(x, \dot{x}, 0,0,0,0, t),
$$

which is identical to equation (19), which we started with.

A constraint like $z(t)=0$ is a special case of constraint whose general form is

$$
f(x, y, z, t)=0
$$

If we have a system of $n$ particles described by the $3 n$ coordinates $x_{i}, y_{i}, z_{i}, i=$ $1,2, \ldots, n$, then a constraint of the form

$$
f\left(x_{1}, y_{1}, z_{1}, x_{2}, \ldots, x_{n}, y_{n}, z_{n}, t\right)=0
$$

or a constraint which can be reduced to this form, is called a holonomic constraint. If the holonomic constraint does not have time explicitly in it, it is called a scleronomic constraint; else it is called rheonomic, terms from the Greek, which means rigid and flowing, respectively. Notably, in a holonomic constraint like equation (24) velocities do not directly appear. Constraints are placed on the velocities of the system of particles. This is because the holonomic constraint must be satisfied at all times. 
Example of an holonomic constraint: Consider a pendulum of constant length $L$. Let the coordinates of the bob of the pendulum be $(x, y, z)$. Then the constraint on the motion of the pendulum bob is that

$$
x^{2}+y^{2}+z^{2}=L^{2} .
$$

The configuration space is 3 -dimensional. This is a scleronomic constraint and implies that infinitesimal configuration changes must satisfy the relation

$$
x d x+y d y+z d z=0 .
$$

Differentiating equation (26) with respect to time yields a constraint on the velocity of the pendulum given by

$$
x \dot{x}+y \dot{y}+z \dot{z}=0,
$$

and a further differentiation gives

$$
x \ddot{x}+y \ddot{y}+z \ddot{z}=-\left(\dot{x}^{2}+\dot{y}^{2}+\dot{z}^{2}\right),
$$

which can be put in matrix form as

$$
\left[\begin{array}{lll}
x & y & z
\end{array}\right]\left[\begin{array}{c}
\ddot{x} \\
\ddot{y} \\
\ddot{z}
\end{array}\right]=-\left(\dot{x}^{2}+\dot{y}^{2}+\dot{z}^{2}\right) .
$$

Though started with a 3-dimensional configuration space, it is notable that the bob cannot access any point in this 3-dimensional space while still satisfying the constraint (25). This constraint has actually limited the accessible space of configurations simply to the 3 -dimensional surface of the sphere $x^{2}+y^{2}+z^{2}=$ $L^{2}$.

Nonholonomic Constraints: Any constraint that cannot be put in the form of the equation (24) is a nonholonomic constraint. For instance, if a particle rests on a horizontal surface, then with the Z-direction pointing upwards and normal to the surface, we must have

$$
z(t) \geq 0
$$


Such inequality constraint is nonholonomic. Nonholonomic constraints are often categorized as rheonomic and scleronomic, just like holonomic constraint, according to whether they do, or they do not, explicitly depend on the time. An important class of nonholonomic equality constraints is represented by the equation

$$
\sum_{j=1}^{3 n} d_{i j}(x, t) d x_{j}+g_{i}(x, t) d t=0, \quad i=1,2, \ldots r,
$$

where the system of equations (31) does not possess any integrals. Equations in this form are defined as Pfaffian forms. This is the nonintegrable Pfaffian form that leads to nonholonomic constraints. These constraints prescribe restrictions on the infinitesimal displacements of the system; our inability to integrate them indicates that we cannot find the corresponding restrictions on the finite displacements of the system.

Whether the Pfaffian forms are integrable or nonintegrable, they can always be differentiated, provided the functions $d_{i} j(x, t)$ and $g_{i}(x, t)$ are sufficiently smooth, to yield the set of $m=h+r$ equations

$$
\begin{array}{r}
\sum_{j=1}^{3 n} d_{i j}(x, t) \ddot{x}_{j}+\sum_{j=1}^{3 n} \sum_{k=1}^{3 n} \frac{\partial d_{i j}(x, t)}{\partial x_{k}} \dot{x}_{k} \dot{x}_{j}+\sum_{j=1}^{3 n} \frac{\partial d_{i j}(x, t)}{\partial t} \dot{x}_{j}+ \\
\sum_{k=1}^{3 n} \frac{\partial g_{i}(x, t)}{\partial x_{k}} \dot{x}_{k}+\frac{\partial g_{i}(x, t)}{\partial t}=0, \quad i=1,2, \ldots m .
\end{array}
$$

These $m$ equations can be expressed in matrix form

$$
A(x, t) \ddot{x}=b(x, \dot{x}, t),
$$

where the $i$-jth element of the $m$ by $3 n$ matrix $A$ is $d_{i j}(x, t)$, and $b_{i}(x, \dot{x}, t)$, the ith row element of the $m$-vector $\mathrm{b}$, is given by

$$
\begin{array}{r}
b_{i}(x, \dot{(x)}, t)=-\sum_{j=1}^{3 n} \sum_{k=1}^{3 n} \frac{\partial d_{i j}(x, t)}{\partial x_{k}} \dot{x}_{k} \dot{x}_{j}-\sum_{j=1}^{3 n} \frac{\partial d_{i j}(x, t)}{\partial t} \dot{x}_{j} \\
-\sum_{k=1}^{3 n} \frac{\partial g_{i}(x, t)}{\partial x_{k}} \dot{x}_{k} \frac{\partial g_{i}(x, t)}{\partial t} .
\end{array}
$$


The $3 n$ acceleration vector in $(33)$ is $\ddot{x}=\left[\ddot{x}_{1}, \ddot{x}_{2}, \ddot{x}_{3}, \ldots, \ddot{x}_{3 n-1}, \ddot{x}_{3 n}\right]^{T}$.

Notice that for the holonomic and nonholonomic Pfaffian equality constraints, the matrix $A$ depends, in general, on $x$ and $t$. The vector $b$ depends on $x, \dot{x}$ and $t$.

Example of a nonholonomic constraint: Consider a constraint on a particle of the form:

$$
\dot{x}=\dot{z}^{2} \dot{y}
$$

The equation is nonintegrable. This nonholonomic constraint can be expressed in the form of equation (33) by differentiating with respect to time, to give,

$$
\ddot{x}=z^{2} \ddot{y}+2 z \dot{z} \dot{y},
$$

or in the matrix form $A(x, t) \ddot{x}=b(x, \dot{x}, t)$ as

$$
\left[\begin{array}{lll}
1 & -z^{2} & 0
\end{array}\right]\left[\begin{array}{c}
\ddot{x} \\
\ddot{y} \\
\ddot{z}
\end{array}\right]=2 z \dot{z} \dot{y} .
$$

The matrix $\mathrm{A}$ is 1 by 3 , and the vector $\mathrm{b}$ is a scalar.

\subsubsection{Gauss's Principle}

Consider a system of $n$ particles of masses $m_{1}, m_{2}, \ldots, m_{n}$. Let the 3-vector $x_{i}=\left[x_{i}, y_{i}, z_{i}\right]^{T}$ represent the position of the ith particle in a rectangular inertial frame of reference. It is assumed that the ith particle is subjected to the given impressed force $F_{i}(t)$, so that its acceleration, were no constraints present, would be given by the 3 -vector $a_{i}=\frac{1}{m_{i}} F_{i}(t)$. The three components of the vector $a_{i}$ would correspond to the accelerations of the ith particle in the three mutually perpendicular coordinate directions, and the three components of $F_{i}$ would be the forces on that particles in those corresponding three directions. It is also assumed that the particles are constrained through certain interconnections. Our goal is to determine the actual accelerations of the particles at any given time $t$, as a result of the given impressed forces and the constraints, given that we 
know the position and velocity of each particle at time t. Thus the equations of motion, where there are no constraints on the particles of the system, can be simply written as

$$
M \ddot{a}=F(x(t), \dot{x}(t), t)
$$

where the $3 n$-vector $\mathrm{F}$ of the given impressed forces is obtained by stacking the known impressed forces $F_{i}(t)$ acting on each of the particles of the system, so that $F(t)=\left[F_{1}^{T}, F_{2}^{T}, \ldots, F_{n}^{T}\right]^{T}$; the $3 n$-vector a is obtained by stacking the corresponding vector $a_{i}(t)$ of each of the particles so that $a(t)=\left[a_{1}^{T}, a_{2}^{T}, \ldots, a_{n}^{T}\right]^{T}$; and the $3 n$ by $3 n$ matrix $M$ is, as usual, diagonal with the masses located on the main diagonal in sets of three do that $M=\operatorname{Diag}\left\{m_{1}, m_{1}, m_{1}, m_{2}, \ldots, m_{n}, m_{n}, m_{n}\right\}$. Similarly, there is the $3 n$-vector of position given by $x(t)=\left[x_{1}^{T}, x_{2}^{T}, \ldots, x_{n}^{T}\right]^{T}$. When said that the $F_{i}$ forces are given, it means that they are known functions of $x, \dot{x}$ and $t$.

In the presence of constraints, the accelerations of the particles at time $t$ will differ from $a(t)$ and this acceleration is denoted by the $3 n$-vector $\ddot{x}(t)$, which is obtained by stacking the corresponding accelerations of each particle, so that $\ddot{x}(t)=\left[\ddot{x}_{1}^{T}, \ddot{x}_{2}^{T}, \ldots, \ddot{x}_{n}^{T}\right]^{T}$. Is assumed that $x$ and $\dot{x}$ are known at time $t$ and also the entire impressed force vector $F(x, \dot{x}, t)$ at that time. Furthermore, is also assumed that both vectors $x$ and $\dot{x}$ are compatible with the given constraints. We note that the matrix $M$ consists of positive entries down the diagonal and is therefore positive definite.

Gauss's principle asserts that among all the accelerations that the system can have at time $t$, which are compatible with the constraints, the ones that actually materialize are those that minimize the following quantity:

$$
G(\ddot{x})=(\ddot{x}-a)^{T} M(\ddot{x}-a)=\left(M^{1 / 2} \ddot{x}-M^{1 / 2} a\right)^{T}\left(M^{1 / 2} \ddot{x}-M^{1 / 2} a\right) .
$$

Shall be referred for this scalar, for short, as the Gaussian, G. This principle is applicable to any type of kinematical constraint that the system may be subjected to.

Is noted that the quantity $\Delta \ddot{x}=\ddot{x}-a$ is simply the deviation of the acceleration of the constrained system from what it would be, had there been no constraints on it. Thus the quantity $\mathrm{G}$ can be thought of as the square of the normalizes length 
of the vector $\Delta \ddot{x}$, normalized with respect to the matrix $M$. Clearly, when there are no constraints, the minimum of $G(\ddot{x})$ is achieved when $\ddot{x}=a$, the acceleration of the system when it is unconstrained, and $\Delta \ddot{x}=0$.

Will only be considered those constraints which can be expressed as linear equality relations between the accelerations of the particles of the system. Thus the constraints that will be dealt with in this project will be of the standard form

$$
A(x, \dot{x}, t) \ddot{x}=b(x, \dot{x}, t)
$$

where the matrix $A$ is $m$ by $3 n$ and the vector $b$ is an $m$-vector. Note that the inequality constraints like those expressed by equation (30) cannot be put into this form.

\subsubsection{Fundamental Equation}

Is asserted that at each instant of time $t$, the actual acceleration $3 n$-vector $\ddot{x}$ of the system of $n$ particles, in the presence of the constraints which have been expressed by equation (40), is given by

$$
\ddot{x}=a+M^{-1 / 2}\left(A M^{-1 / 2}\right)^{+}(b-A a),
$$

where $\left(A M^{-1 / 2}\right)^{+}$is the unique MP-inverse of the Constraint Matrix $A M^{-1 / 2}$.

To verify the equation, is shown that the acceleration given by equation (41) satisfy equation (40), then is shown that of all the accelerations vectors which satisfy equation (40), it is the unique vector that minimizes the Gaussian G, which has been defined in equation (39). If the acceleration vector $\ddot{x}$ satisfy these two conditions, Gauss's principle then assures that this accelerations is then the correct acceleration of the particles contituting the constrained system.

Note that the equation (40) can be expressed as

$$
A M^{-1 / 2}\left(M^{1 / 2} \ddot{x}\right)=A M^{-1 / 2}(y)=b
$$

So that this equation can be considered consistent, is required, by equation (14), or better $A A^{+} b=b$, that

$$
A M^{-1 / 2}\left(M^{-1 / 2}\right)^{+} b=b
$$


Substituting the expression for $\ddot{x}$ given in equation (41) into the left hand side of equation (40), we get

$$
\begin{aligned}
A \ddot{x} & =A a+A M^{-1 / 2}\left(A M^{-1 / 2}\right)^{+}(b-A a) \\
& =\left[I-A M^{-1 / 2}\left(A M^{-1 / 2}\right)^{+}\right] A a+A M^{-1 / 2}\left(A M^{-1 / 2}\right)^{+} b \\
& =\left[I-A M^{-1 / 2}\left(A M^{-1 / 2}\right)^{+}\right]\left(A M^{-1 / 2}\right) M^{1 / 2} a+A M^{-1 / 2}\left(A M^{-1 / 2}\right)^{+} b
\end{aligned}
$$

Considering the MP conditions, the first term on the right hand side of the last expression above vanishes, se we get

$$
A \ddot{x}=A M^{-1 / 2}\left(A M^{-1 / 2}\right)^{+} b=b
$$

where the last equality follows because of equation (43). Hence the acceleration $\ddot{x}$ as defined by equation (41) satisfies the constraint equation (40).

It is notable that the algebra can be greatly simplified if the matrix $M=m I$, and using the equation (7) we get

$$
M^{-1 / 2}\left(A M^{-1 / 2}\right)^{+}=m^{-1 / 2} I\left(m^{-1 / 2} A I\right)^{+}=A^{+}
$$

When the matrix $M=m I$, the fundamental equation (41) can be simplified as

$$
\ddot{x}=a+A^{+}(b-A a)
$$

\subsubsection{The Force of Constraint}

The presence of a constraint causes the acceleration of a system at every instant of time to deviate from that which it would have had, had there been no constraints. This deviation in acceleration of the constrained system is brought by a force that is exerted in the system by the fact that the unconstrained system must now further satisfy the constraints. Consider an instant of time $t$. The equation of motion of the unconstrained system, given by

$$
M a=F(t),
$$

where, the vector $F$ consists on the known impressed forces on the system, can be compared with the equation of the constraint system, given by 


$$
M \ddot{x}=M a+M^{1 / 2}\left(A M^{-1 / 2}\right)^{+}(b-A a) .
$$

Using equation (48) in equation (49), the motion of the constrained system can alternately be described as

$$
M \ddot{x}=F(t)+M^{1 / 2}\left(A M^{-1 / 2}\right)^{+}(b-A a)=F(t)+F^{c}(t) .
$$

Thus, at each instant of time $t$, the constraint system is subjected to an additional "constraint force" $F^{c}(t)$ given by

$$
F^{c}(t)=M^{1 / 2}\left(A M^{-1 / 2}\right)^{+}(b-A a) .
$$

It is this additional force that causes the acceleration of the system at time $t$ to change from its unconstrained value $a(t)$ to its constraint value of $\ddot{x}(t)$.

Nowhere in the previous explanation, were required that the set of constraint equation be linearly independent. Hence equation (41) and (51) are valid even when the constraint equations are linearly dependent. Often in complex systems it becomes difficult to ascertain which of the constraints are linear dependent. However, this difficulty poses no problem to the approach used in this project.

As before, when the matrix $M$ is a constant diagonal matrix so that $M=m I$, then the equation (51), simplifies to

$$
F^{c}(t)=m A^{+}(b-A a)
$$




\section{Example of constrained motions}

\subsection{Example 1: Scleronomic constraint}

Consider a constraint

$$
\dot{x}+2 y \dot{y}+\dot{z}=0
$$

It shows that equation (53) is scleronomic. Take the integral of equation (53) with respect to time $t$, then we reformulate the equation as

$$
x+y^{2}+z+C=0,
$$

where $C$ is a constant. According to what have been said in this project, constraints in which time is not explicitly present are called scleronomic. Therefore, the equation (53) is not only holonomic but also scleronomic.

\subsection{Example 2: Rheonomic constraint}

Consider a constraint

$$
\dot{x}+2 y \dot{y}+\dot{z}=1
$$

It shows that equation (55) is rheonomic. Take the integral of equation (55) with respect to time $t$, then we reformulate the equation as

$$
x+y^{2}+z-t+C=0,
$$

where $C$ is a constant. According to what have been said in this project, constraints in which time explicitly enters into the constraint equation are called rheonomic. Therefore, the equation (55) is not only holonomic but also rheonomic.

According with what seen through this project, is known that equation (53) is not only holonomic but also rheonomic.

\subsection{Example 3: Fundamental Equation Applied 1}

Consider this example [1] of the coupled Duffing's oscillator shown in figure 1 which is subjected to the constraint 


$$
x_{1}(t)-x_{2}(t)=A_{0} \exp (-\alpha t) \sin (\omega t) .
$$

where $A_{0}, \alpha$ and $\omega$ are given constants.

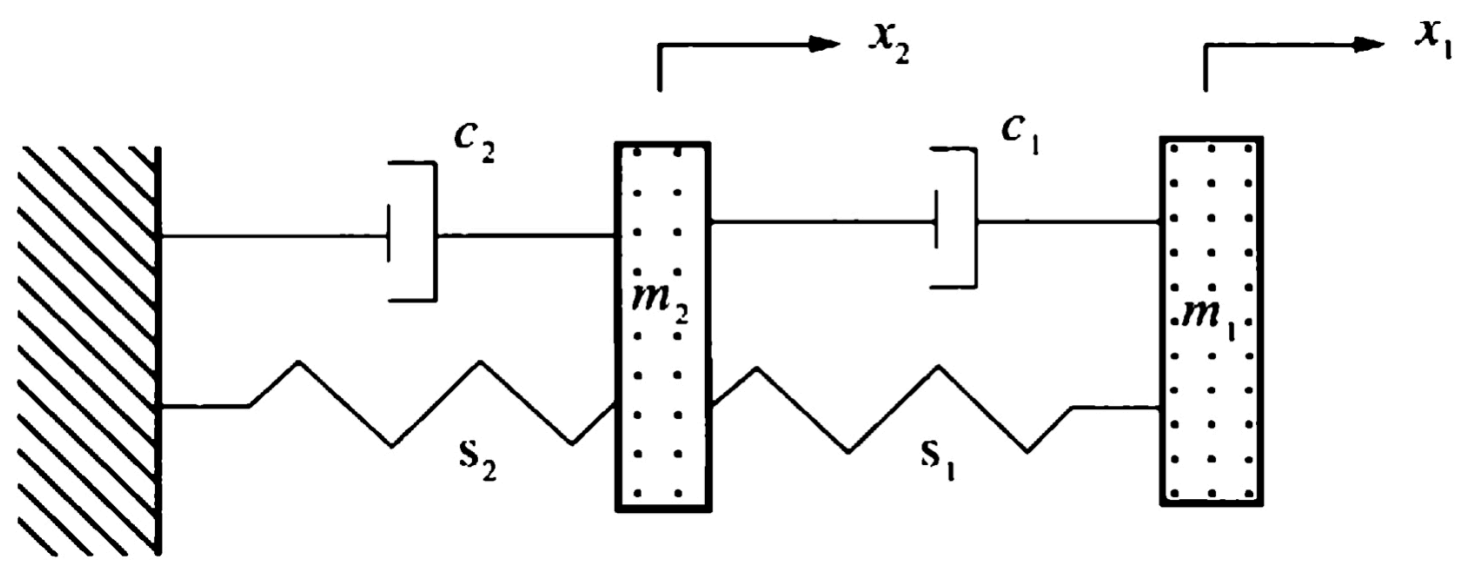

Figure 1: Coupled Duffing's Oscillator

The two nonlinear springs $s_{1}$ and $s_{2}$ exert forces denoted by

$$
f_{i}=k_{i} u_{i}+k_{i}^{(n l)} u_{i}^{3}, \quad i=1,2,
$$

where $u_{i}$ denotes the extension of the ith spring. The second term involving $k_{i}^{n l}$ on the right hand side of equation (58) indicates that the spring force has a cubic nonlinearity, denoted by the $(n l)$. The damping elements, represented by $f_{i}^{c}$, are linear viscous dampers exerting forces

$$
f_{i}^{c}=c_{i}(\dot{u})_{i}, \quad i=1,2 .
$$

The equation of motion of the unconstrained system may be written as

$$
M \ddot{x}=-\left[K x+C \dot{x}+g^{(n l)}\right]=F,
$$

where

$$
x=\left[\begin{array}{ll}
x_{1} & x_{2}
\end{array}\right] \text { and } M=\left[\begin{array}{cc}
m_{1} & 0 \\
0 & m_{2}
\end{array}\right]
$$

The matrices 


$$
K=\left[\begin{array}{cc}
k_{1} & -k_{1} \\
-k_{1} & k_{1}+k_{2}
\end{array}\right] \text { and } C=\left[\begin{array}{cc}
c_{1} & -c_{1} \\
-c_{1} & c_{1}+c_{2}
\end{array}\right]
$$

and the vector $g^{(n l)}$, which appear due to the non-linear spring forces, is given by

$$
g^{(n l)}=\left[\begin{array}{c}
k_{1}^{(n l)}\left(x_{1}-x_{2}\right)^{3} \\
k_{2}^{(n l)} x_{2}^{3}-k_{1}^{(n l)}\left(x_{1}-x_{2}\right)^{3}
\end{array}\right] .
$$

The acceleration of the unconstrained system is given by

$$
a(t)=\left[\begin{array}{l}
a_{1}(t) \\
a_{2}(t)
\end{array}\right]=M^{-1} F .
$$

Differentiating equation (57) twice, we get, the constraint equation

$$
\ddot{x}_{1}(t)-\ddot{x}_{2}(t)=-A_{0} \exp (-\alpha t)\left\{\omega^{2} \sin (\omega t)+2 \omega \alpha \cos (\omega t)-\alpha^{2} \sin (\omega t)\right\}=b(t) .
$$

Hence the matrix $A=[1-1]$ and $b$ is a scalar which equals $b(t)$. We then obtain

$$
A M^{-1 / 2}=\left[\begin{array}{ll}
m_{1}^{-1 / 2} & -m_{2}^{-1 / 2}
\end{array}\right] \text { and }\left(A M^{-1 / 2}\right)^{+}=\frac{1}{m_{1}^{-1}+m_{2}^{-1}}\left[\begin{array}{c}
m_{1}^{-1 / 2} \\
-m_{2}^{-1 / 2}
\end{array}\right]
$$

and the equation of motion of the constrained system becomes

$$
\ddot{x}=\left[\begin{array}{l}
a_{1}(t) \\
a_{2}(t)
\end{array}\right]+\frac{m_{1} m_{2}}{m_{1}+m_{2}}\left[\begin{array}{c}
m_{1}^{-1} \\
-m_{2}^{-1}
\end{array}\right]\left\{b(t)-a_{1}(t)+a_{2}(t)\right\} .
$$

Notice that the effect of the constraint is encapsulated in the second term on the right hand side of equation (67). The force required to be applied to the two masses to "guide" the system so that it satisfies the constraint is explicity given by

$$
F^{c}=\left[\begin{array}{l}
F_{1}^{c} \\
F_{2}^{c}
\end{array}\right]=\frac{m_{1} m_{2}}{m_{1}+m_{2}}\left[\begin{array}{c}
1 \\
-1
\end{array}\right]\left\{b(t)-a_{1}(t)+a_{2}(t)\right\} .
$$


Some numerical results are obtained from integrated equation (68) using the software MATLAB. The 4th- and 5th-order Runge-Kutta method is used for the time integration scheme with a time-step equal to $10^{-6} \mathrm{~s}$ in a range of $[0,5]$ seconds. The computer program can be found at the appendix.

The parameter values describing the system are $m_{1}=2, m_{2}=1, k_{1}=10, k_{2}=$ $12, k_{1}^{(n l)}=1, k_{2}^{(n l)}=2, c_{1}=0.1, c_{2}=0.15$. The parameters describing the constraint of equation (57) are $A_{0}=1, \omega=2 \pi$ and $\alpha=1$. The initial conditions chosen are:

$$
\begin{gathered}
x_{1}(0)=x_{2}(0)=1, \text { and }, \\
\dot{x}_{1}(0)=A_{0} \omega+\dot{x}_{2}(0), \quad \dot{x}_{2}(0)=2 .
\end{gathered}
$$

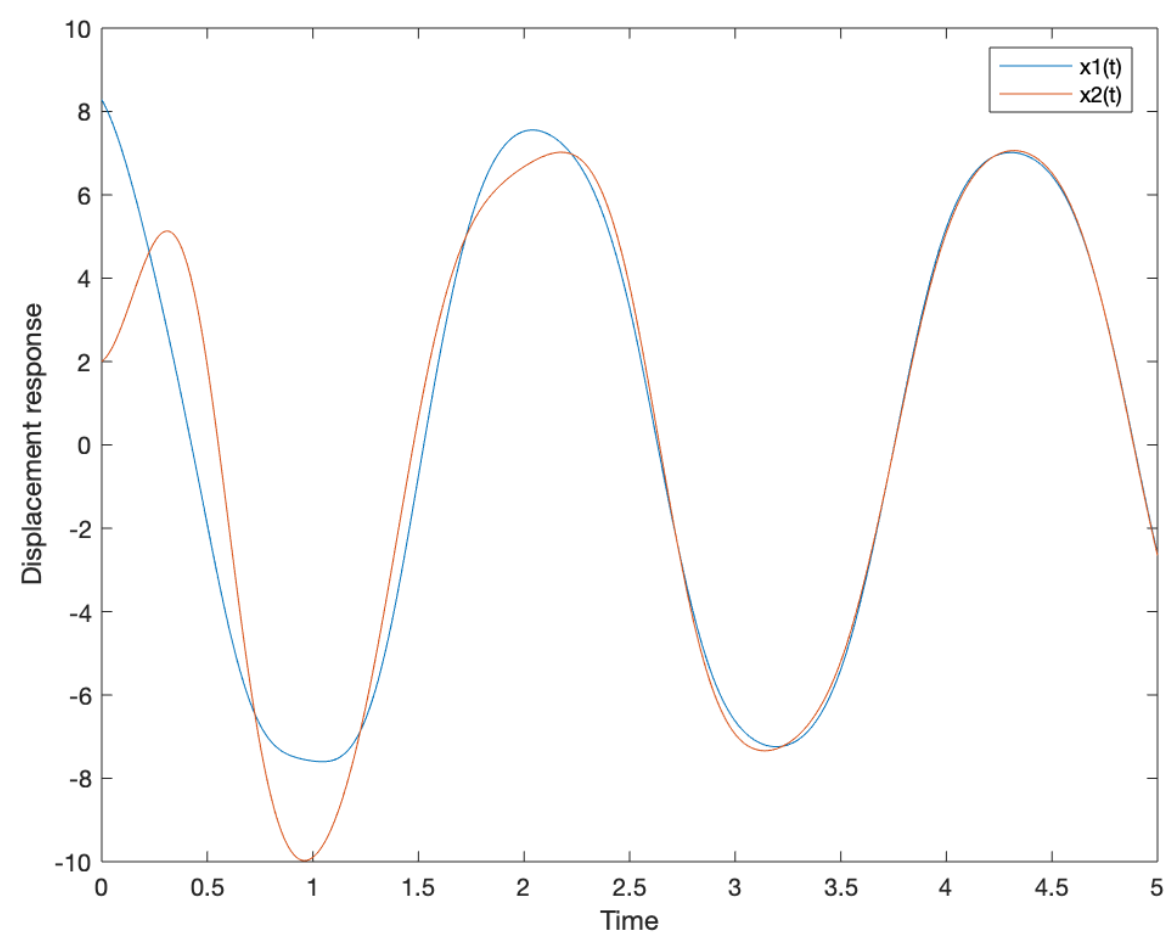

Figure 2: Response of the system without constraint forces

The figure 2 shows the response of the system without the constraint of equation (57) and figure 3 with the constraint.

Figure 4 shows the extent to which the constraint is satisfied by plotting the difference $\left[x_{1}(t)-x_{2}(t)\right]$ versus time $t$. Figure 4 shows this difference when the 


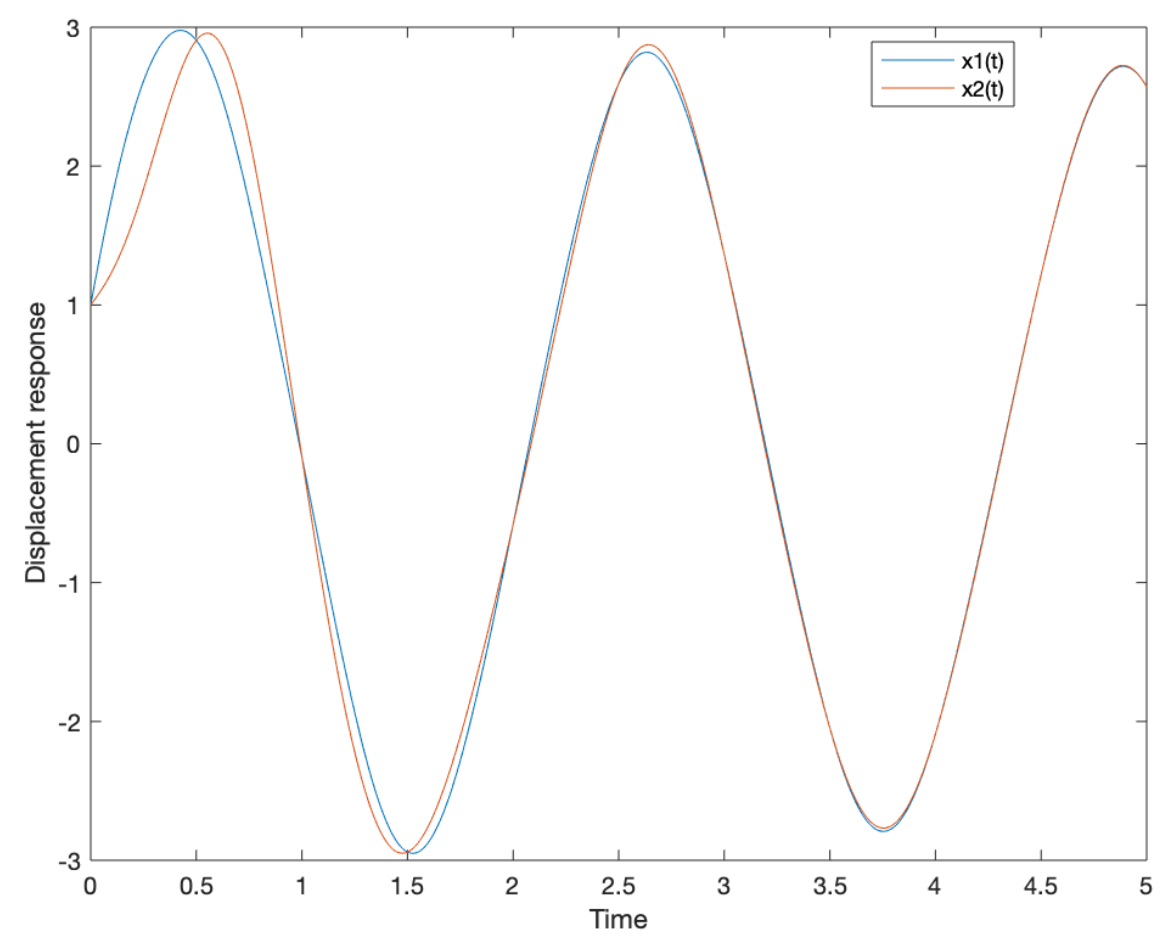

Figure 3: Response of the system with constraint forces

constraint is imposed. In this figure, is also shown the function $A_{0} \exp (-\alpha t) \sin (\omega t)$ plotted by a dashed line for comparison. To the scale plotted, the difference between the solid line and the dashed line cannot be discerned.

Figure 5 depicts the forces that need to be applied to the masses so that they are "guided" to follow the constraint of equation (57). The constraint force applied to mass $m_{1}$ is shown by a solid line, and the one applied to mass $m_{2}$ by a dashed line.

Figure 6 shows the numerical error in the integration scheme used, as it is reflected through its ability to keep the constrained satisfied. This numerical error is denoted as error $(t)=x_{1}(t)-x_{2}(t)-A_{0} \exp (-\alpha t) \sin (\omega t)$. The constraints get gradually violated in time, due to the numerical accuracy of the integration scheme. 


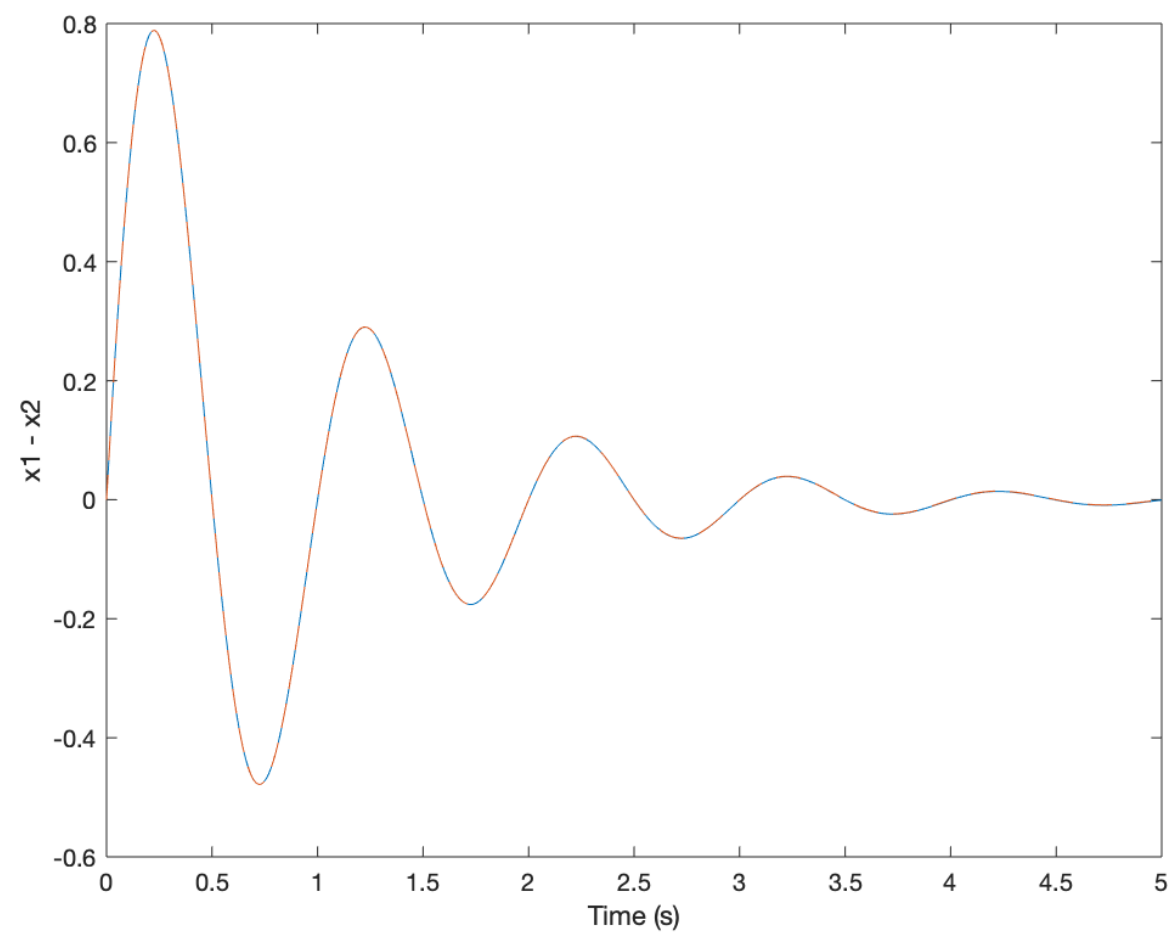

Figure 4: Figure illustrating the effect of the constraint force

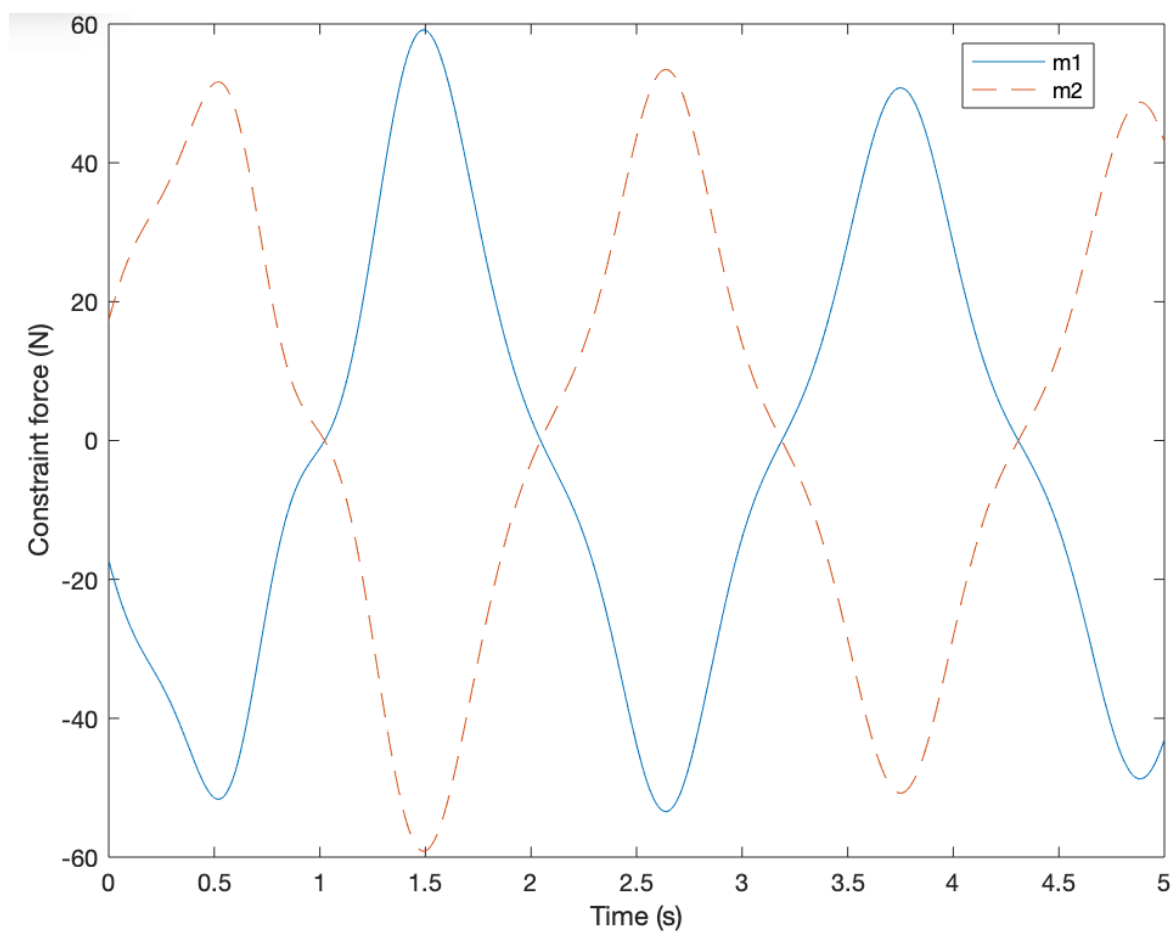

Figure 5: The force of constraint acting on the two masses 


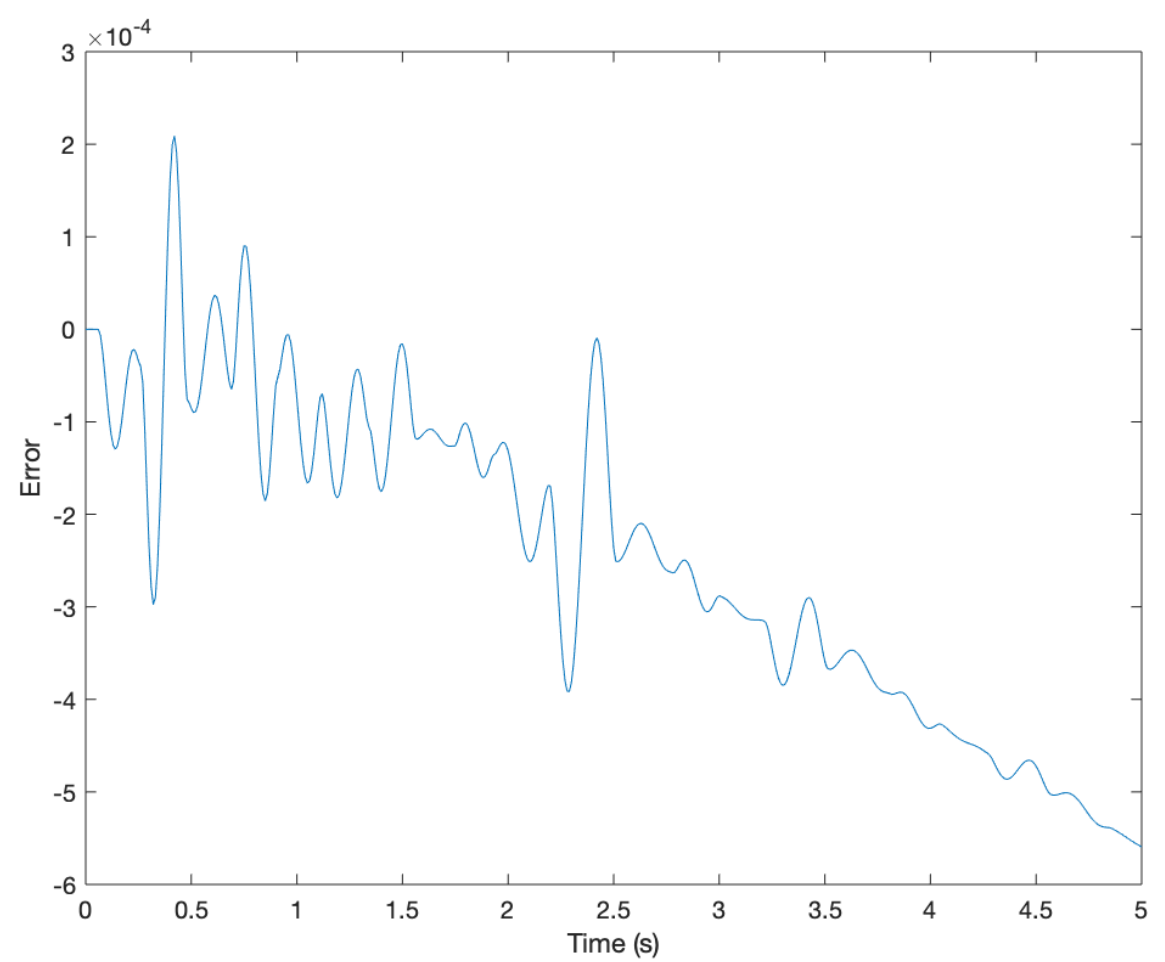

Figure 6: Numerical error due to the accuracy of the integration scheme

\subsection{Example 4: Fundamental Equation Applied 2}

Consider a pendulum bob of unit mass suspended by a weightless rod of length $L_{1}$, which is attached to the origin of the rectangular coordinate system. The bob is also connected to another weightless rod of length $L_{2}$ whose other end is pinned to the point $\left(L_{1}, 0, L_{2}\right)$. Figure 7 represents this pendulum.

The equations describing the constraints can be written as:

$$
\begin{gathered}
x^{2}+y^{2}+z^{2}=L_{2}^{2}, \text { and, } \\
\left(x-L_{1}\right)^{2}+y^{2}+\left(z-L_{2}\right)^{2}=L_{1}^{2} .
\end{gathered}
$$

Differentiating these equations twice, we can rewrite them as

$$
A \ddot{x}=b \text {, }
$$

where, 


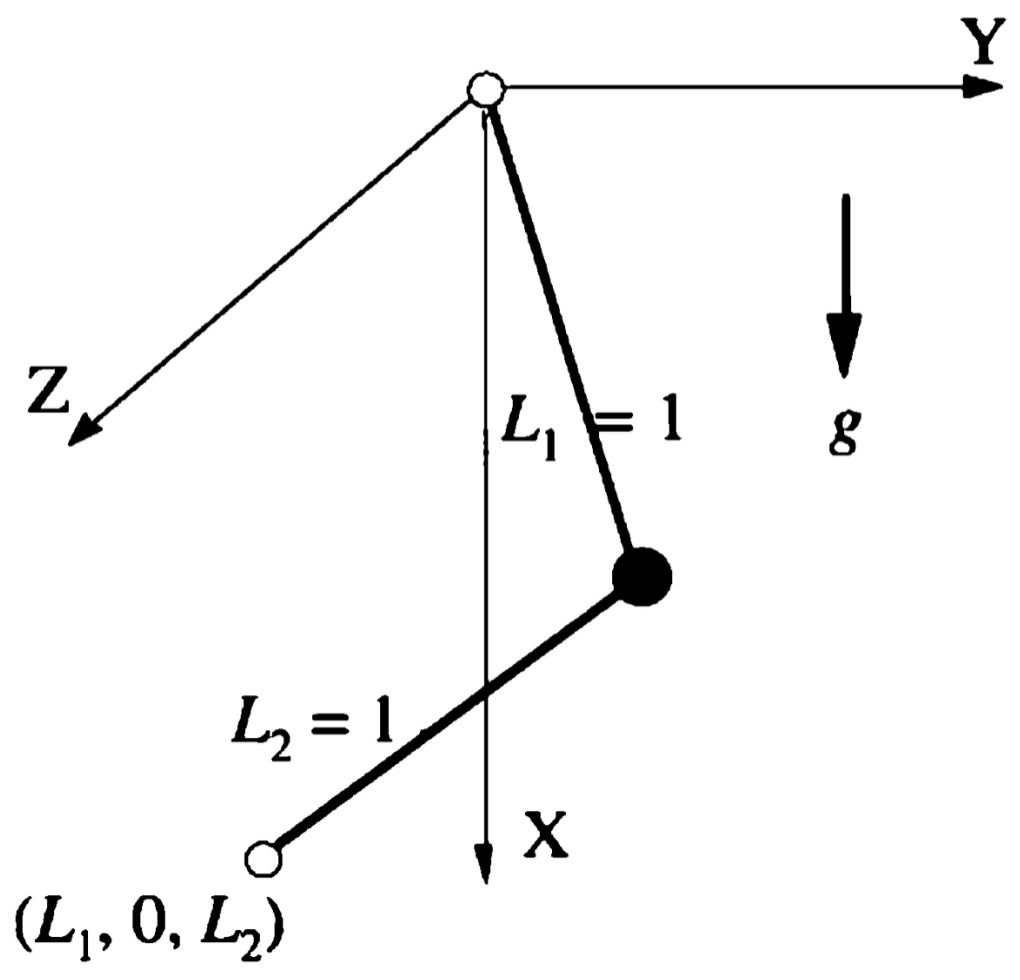

Figure 7: Two-link pendulum

$$
A=\left[\begin{array}{ccc}
x & y & z \\
x-L_{1} & y & z-L_{2}
\end{array}\right], \text { and } b=-\alpha\left[\begin{array}{l}
1 \\
1
\end{array}\right]
$$

with $\alpha=\left(\dot{x}^{2}+\dot{y}^{2}+\dot{z}^{2}\right)$. The matrix $M$ is a three-dimensional matrix, and the acceleration of the unconstrained system is given by $a=\left[\begin{array}{lll}g & 0 & 0\end{array}\right]^{T}$. Using equation (12), we obtain

$$
\begin{array}{r}
B^{+}=\left(A M^{-1 / 2}\right)^{+}=B^{T}\left(B B^{T}\right)^{-1} \\
=\frac{1}{\Delta}\left[\begin{array}{cc}
L_{2}^{2} x+L_{1} y^{2}+L_{1}^{2} z^{2}-L_{1} L_{2} z-L_{2} x z & L_{2} x z-L_{1} y^{2}-L_{1} z^{2} \\
y\left(L_{1}^{2}+L_{2}^{2}-L_{1} x-L_{2} z\right) & y\left(L_{1} x+L_{2} z\right) \\
L_{2} x^{2}+L_{2} y^{2}-L_{1} L_{2} x+L_{1}^{2} z-L_{1} x z & L_{1} x z-L_{2} x^{2}-L_{2} y^{2}
\end{array}\right]
\end{array}
$$

where $\Delta=\left[L_{2}^{2} x^{2}+\left(L_{1}^{2}+L_{2}^{2}\right) y^{2}-2 L_{1} L_{2} x z+L_{1}^{2} z^{2}\right]$. The vector

$$
b-A a=\left[\begin{array}{c}
-\alpha-g x \\
-\alpha-g\left(x-L_{1}\right)
\end{array}\right],
$$


so that

$$
\begin{aligned}
& {\left[\begin{array}{c}
\ddot{x} \\
\ddot{y} \\
\ddot{z}
\end{array}\right]=\left[\begin{array}{l}
g \\
0 \\
0
\end{array}\right]+B^{+}\left[\begin{array}{c}
-\alpha-g x \\
-\alpha-g\left(x-L_{1}\right)
\end{array}\right] } \\
= & \frac{1}{\Delta}\left[\begin{array}{c}
-\alpha L_{2}\left(L_{2} x-L_{1} z\right)+g L_{2}^{2} y^{2} \\
-y\left(\alpha L_{1}^{2}+\alpha L_{2}^{2}+g L_{2}^{2} x-g L_{1} L_{2} z\right) \\
\alpha L_{1}\left(L_{2} x-L_{1} z\right)-g L_{1} L_{2} y^{2}
\end{array}\right] .
\end{aligned}
$$

For $L_{1}=L_{2}=1$, equation (77) reduces to

$$
\left[\begin{array}{c}
\ddot{x} \\
\ddot{y} \\
\ddot{z}
\end{array}\right]=\frac{1}{\Delta}\left[\begin{array}{c}
-\alpha(x-z)+g y^{2} \\
-y(2 \alpha+g x-g z) \\
\alpha(x-z)-g y^{2}
\end{array}\right]
$$

with $\Delta=\left[x^{2}+2 y^{2}-2 x z+z^{2}\right]$.

Using the initial conditions $x(0)=L_{1}, y(0)=0, z(0)=0, \dot{x}(0)=0, \dot{y}(0)=$ $4, \dot{z}(0)=0$, which satisfies the constraints described by equations (71) and (72) with $L_{1}=L_{2}=1$ is now possible to numerically integrate equation (78). It will be shown the results of this integration with $g=10$ units.

Notably, if the second constraint, represented by the equation (71), were absent, the motion of the pendulum would have been constrained to lie, for all time, in the $X Y$-plane. However, constraint of equation (71) causes the bob to move out in the $Z$-direction causing an out-of-plane motion.

Some numerical results are obtained using the program Mathematica. Figure 8 shows the displacements $y$ and $z$ of the bob as functions of time.

Figure 9 shows a parametric plot of $y(t)$ versus $z(t)$; time is the parameter that is varied. Notably, in the graph it is possible to see that the motion in the $Z$-direction is significant.

Figure 10 shows a 3 -dimensional plot of the trajectory of the bob in the configuration space. The bob starts at the top in the center of the box in the $Y$-direction where $y=0, x=1$ and $z=0$. It is possible to see how two holonomic constraints reduce the accessible configuration space to just a curve. 

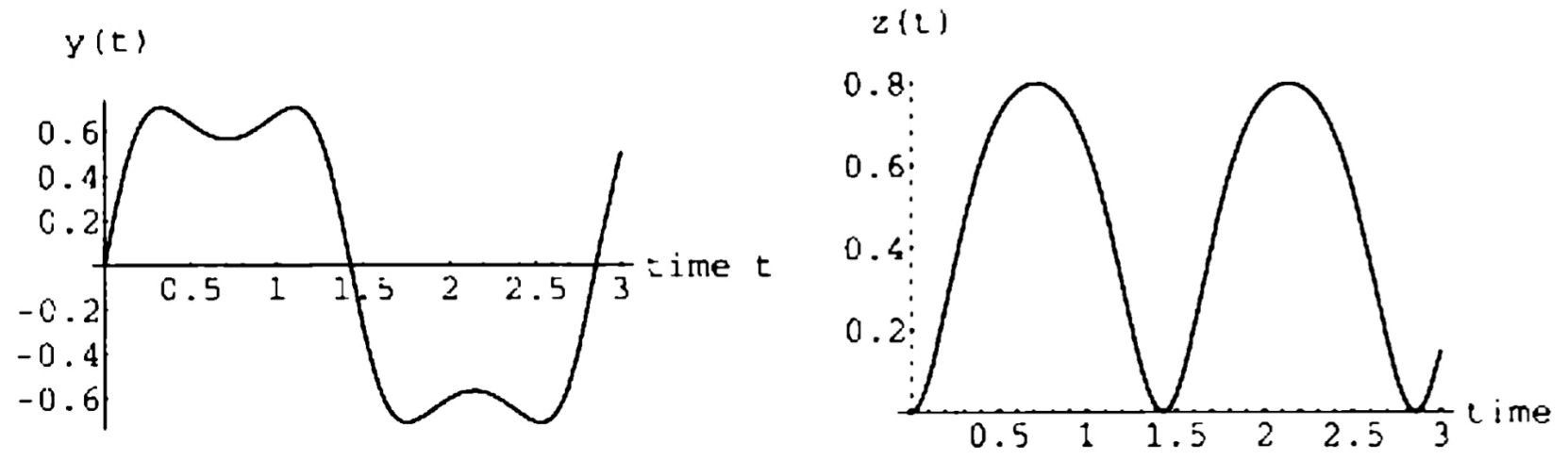

Figure 8: Response of doubly constrained pendulum showing nonlinear behavior $y(t)$

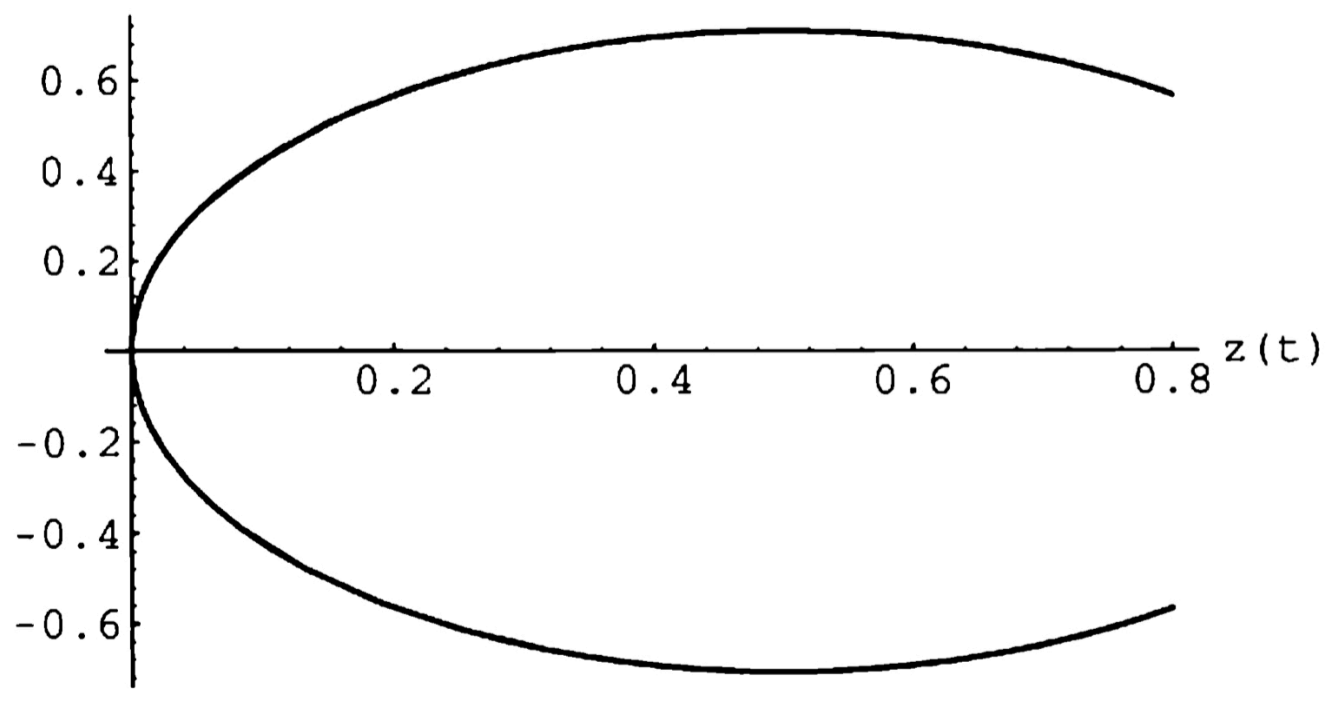

Figure 9: Out-of-plane motion of doubly constrained pendulum 


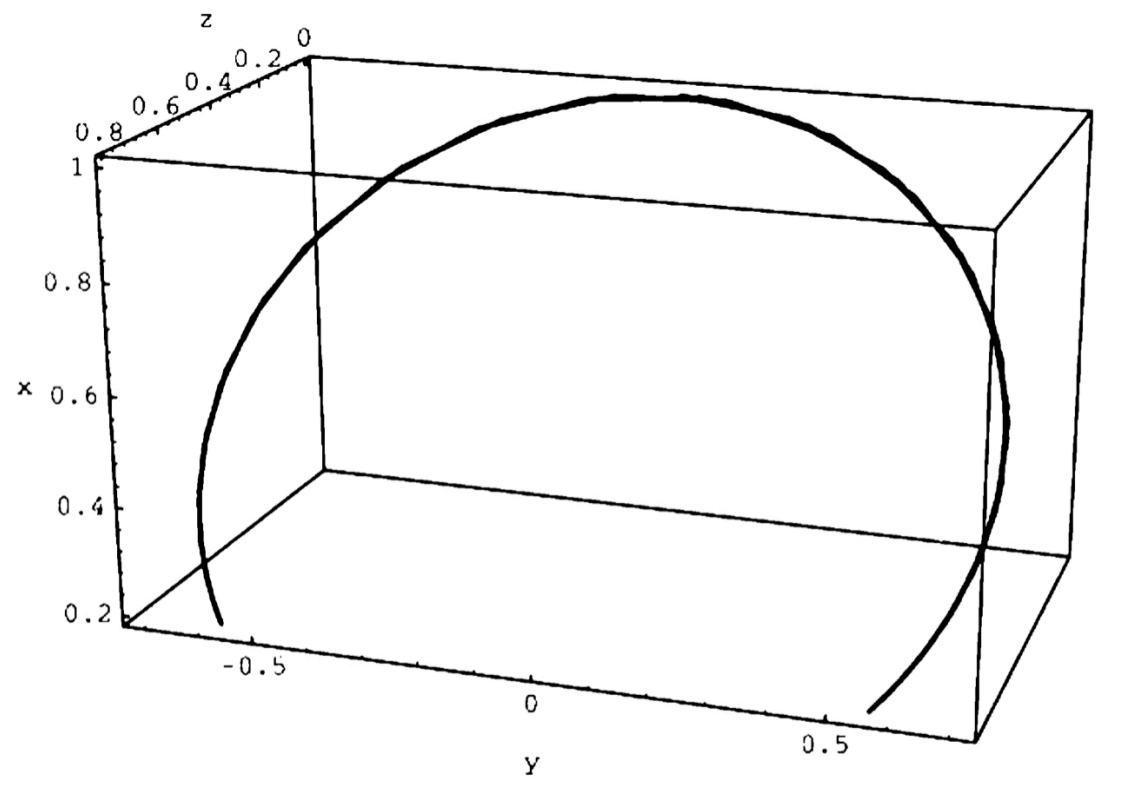

Figure 10: Trajectory of the constrained motion of the pendulum bob 


\section{Conclusions}

The Fundamental equation explicitly provides the acceleration of a constrained system and can be applied to systems with holonomic and/or non-holonomic constraints, as well as constraints that may or may not be ideal. It may be noted that our entire development does not require, nor use, the notion of Lagrange multipliers.

It is also shown that the general, explicit equation of motion obtained in this project reduce to the known and familiar explicit equations of motion when the mass matrices are restricted to being symmetric and positive definite.

The Udwadia-Kalaba method shown in this project proves to be rather straightforward. So far, the Udwadia-Kalaba Equation is the simplest and most comprehensive equation of motion for constrained mechanical systems [12]. Due to its simplicity and closed form, the Udwadia-Kalaba Equation encompasses a wide range of applicability.

The equation studied in this project leads to a practical value in the modelling of complex multi-body systems, where in engineering we expect the equation of motion to be unique.

The Udwadia-Kalaba Equation has greatly contributed to address the complex constrained motion. Since the MP inverse exists in the closed-form constraint force, a high-performance computer is required when the constraints are comprehensive [9].

In general this project has shown some examples of the use of UdwadiaKalaba's equation to complex systems, such as multibody systems subjected to kinematical constraints that also display nonlinear behaviour. Much like Gibbs' and Appell's contribution in the late 19th century to our understanding of constrained mechanical systems with their Gibbs-Appell equation, today Udwadia and Kalaba have brought us an even simpler and more insightful understanding of constrained systems with their Udwadia-Kalaba equation. 


\section{Bibliography}

\section{References}

[1] F E Udwadia, R E Kalaba, Analytical dynamics: A new approach. New York: Cambridge University Press, 2007.

[2] XM. Zhao, YH. Chen, H. Zhao et al, Udwadia-Kalaba Equation for Constrained Mechanical Systems: Formulation and Applications. Chin. J. Mech. Eng. 31, 106 (2018). https://doi.org/10.1186/s10033-018-0310-x

[3] F E Udwadia. Phailaung Phohomsiri, Explicit equations of motion for constraint mechanical systems with singular mass matrices and applications to multi-body dynamics. Proceedings of the Royal Society A: Mathematical, Physical and Engineering Sciences, Royal Society, 2006, 462 (2071), pp.2097 - 2117. <10.1098/rspa.2006.1662>. <hal-01395968>

[4] X. Yang, X. Zhang, Z. Chen, S. Xu, and P. X. Liu, Udwadia-Kalaba Approach for Three Link Manip- ulator Dynamics With Motion Constraints. Vol. 7, 2019

[5] T. Lam, A new approach to mission design based on the fundamental equation of motion. Journal of Aerospace engineering, Vol. 19, No. 2, 2006.

[6] F E Udwadia, R E Kalaba, What is the General Form of the Explicit Equations of Motion for Con-strained Mechanical Systems?. Journal of Applied Mechanics, Vol. 69, No. 3, 2002.

[7] F. E. Udwadia and P. Phohomsiri, Explicit equations of motion for constrained mechanical systems with singular mass matrices and applications to multi-body dynamics. Proceedings of the Royal Society A: Mathematical, Physical and Engineering Sciences, Vol. 462, No. 2071, 2006

[8] J. Baumgarte, Stabilization of constraints and integrals of motion in dynamical systems,. Comput. Methods Appl. Mech. Eng., vol. 1, Jun. 1972.

[9] A.Arabyan and F. Wu, An improved formulation for constrained mechanical systems, . Multibody System Dynamical, vol. 2, no. 1, 1998. 
[10] F. E. Udwadia and P. B. Koganti, Dynamics and control of a multi- body planar pendulum, Nonlinear Dyn., vol. 81, nos. 1-2, 2015.

[11] F. Udwadia and T. Wanichanon, A closed-form approach to tracking control of nonlinear uncertain systems using the fundamental equation, Earth Space, vol. 10, 2012.

[12] F E Udwadia, R E Kalaba, A new perspective on constrained motion, Proc. Roy. Soc. A, Math. Phys. Sci., 1992. 


\section{Appendices}

\section{A Matlab Script}

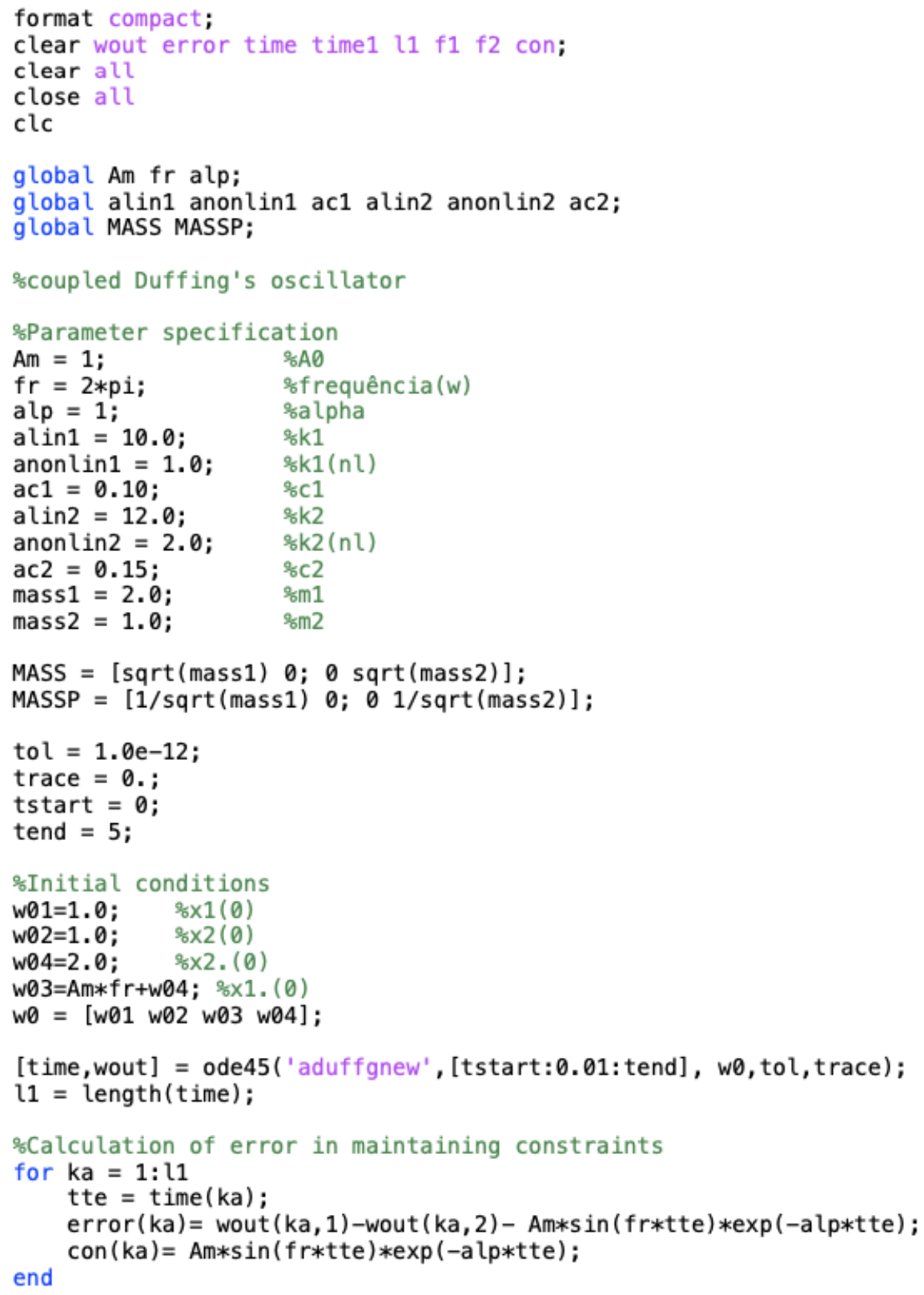




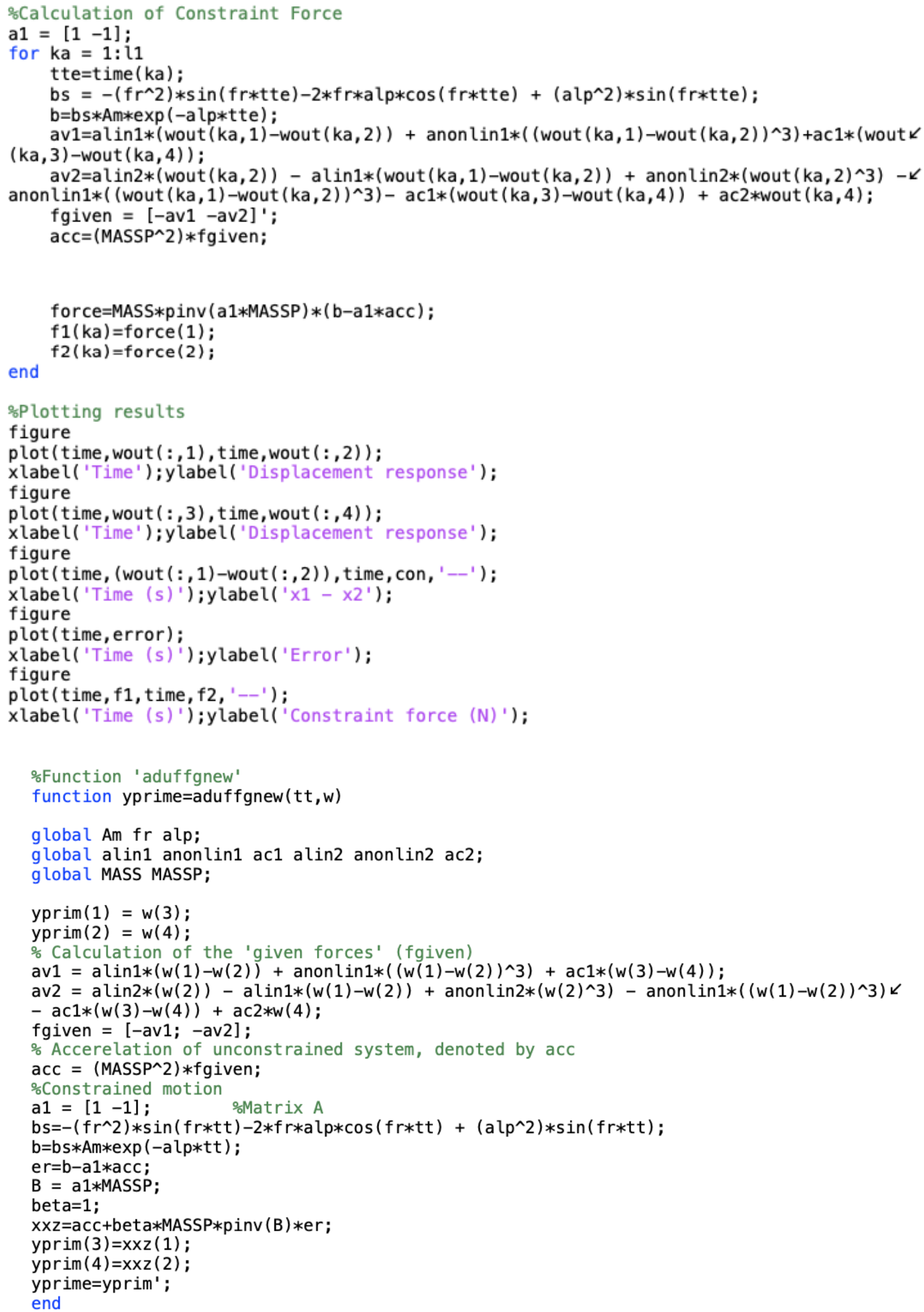




\section{B Mathematica Script}

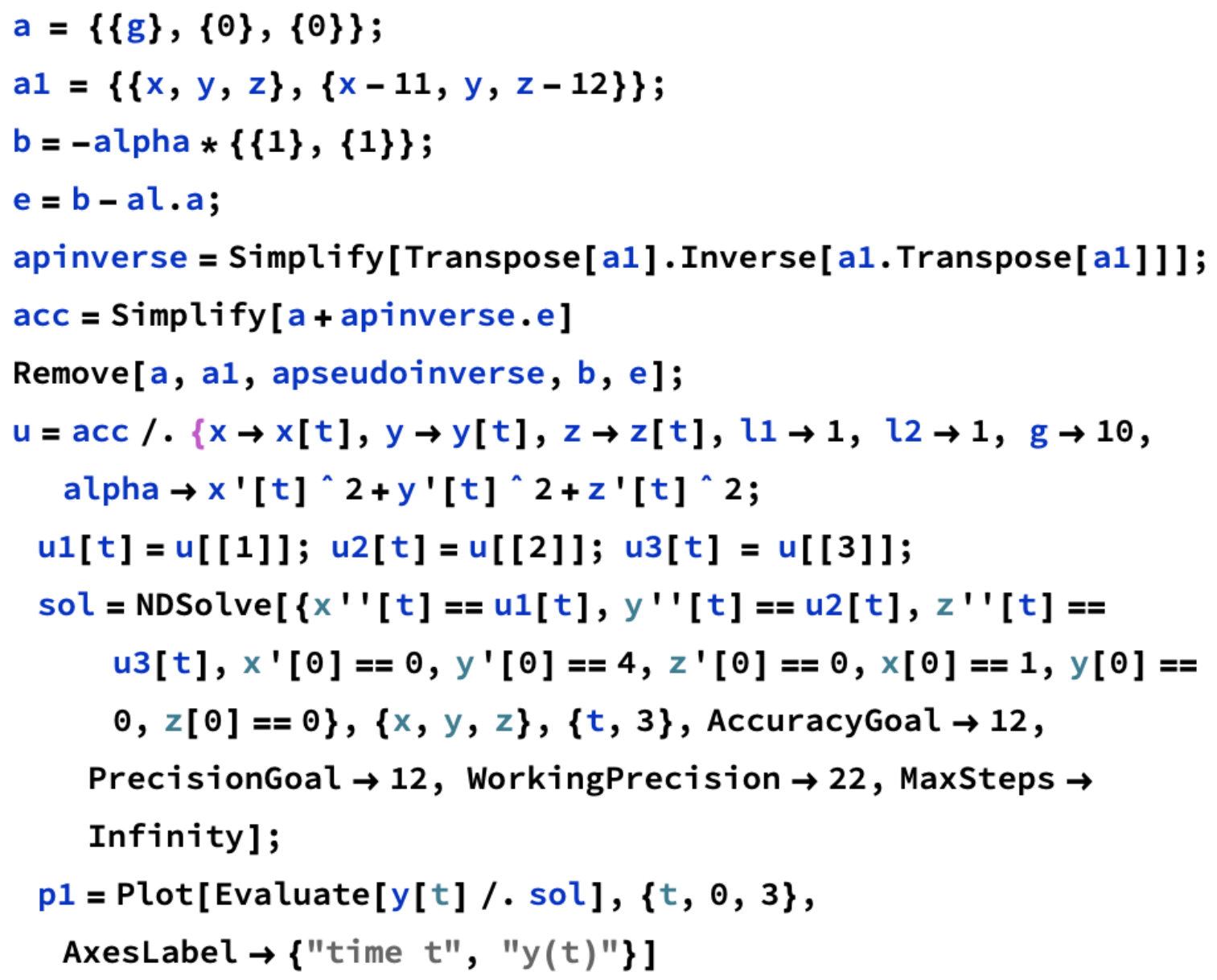

